\title{
Lepton Flavour Violation in the Minimal $S O(10)$ GUT Model and in the Standard Model with additional Heavy Dirac Neutrinos
}

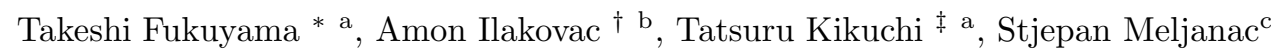

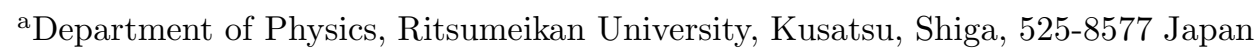 \\ bUniversity of Zagreb, Department of Physics, Bijenička cesta 32, HR-10002 Zagreb, Croatia \\ c'Institut Rudjer Bošković, Bijenička cesta 54, HR-10002 Zagreb, Croatia
}

Lepton flavour violation is considered in two models, Standard Model with additional heavy Dirac neutrinos and minimal supersymmetric standard model (MSSM) with $S O(10)$ theoretical frame and minimal SUGRA (mSUGRA) SUSY-breaking mediation mechanism. The models are briefly explained. The structure of the amplitudes for neutrinoless decays of the lepton into a lepton and semileptonic neutrinoless decays of a lepton is given. The method for identification of meson content in the quark currents is exposed. The comparison of the amplitudes is made showing which of the processes have the largest branching ratios. The numerical results for the dominant decay rates are presented.

\section{Introduction}

Neutrino oscillation data not only show that the neutrinos do have mass and do mix, but these mixings and differences of the squares of the neutrino masses are well determined. Extensions of the Standard Model (SM) are therefore necessary, and any such extension must be able to describe the neutrino data together with all other experimental data which SM describes well. Two of such models are considered here. The simpler of these models, SM extended by heavy (quasi-)Dirac neutrinos [123] (called model A here) has only additional heavy (quasi-) Dirac neutrino fields compared to the SM field content. The second model 456789 (model B) is based on the minimal supersymmetric standard model (MSSM) with heavy right-handed neutrinos [1011, with model parameters determined by the underlying GUT model and through the minimal SUGRA (mSUGRA) SUSY-breaking mediation mechanism [12].

The existence of the neutrino mixings implies that the lepton flavours are violated. An independent confirmation of the lepton flavour violation (LFV) is necessary. The last improvements of the
LFV upper bounds 13] strongly motivate further theoretical investigation of these processes. The structure of the LFV amplitudes and a numerical study of the corresponding decay rates in model A and model $\mathrm{B}$ are the subject of of this paper. The main topic of this paper are semileptonic (SL) neutrinoless LFV decays of charged leptons. The LFV decays of the charged lepton into lepton and photon $\left(\ell \rightarrow \ell^{\prime} \gamma\right)$ are considered also, to determine the bounds on the parameters in the model B.

\section{Models}

The model $\mathrm{A}$ is inspired by a $E_{8} \times E_{8}$ heteroticsuperstring theory. Along with the SM neutrino fields, it contains a number of right-handed neutrino fields and equal number of left-handed $E_{6}$-singlet neutrino fields 1214. The righthanded neutrino fields mix through the Diractype Yukawa couplings with SM neutrino fields and right-handed neutrino fields. The mass matrix has three zero eigenvalues while the other eigenvalues are are large (typically $\sim 1 \mathrm{GeV}$ ). The masses and the mixings of the light neutrinos may be induced through the small Majorana-type 
Yukawa couplings of the left-handed $E_{6}$ singlet neutrino fields 1415. Fitting of the neutrino oscillation data is easily achievable. The small Majorana mass terms promote the heavy Dirac fields to heavy quasi-Dirac fields. The structure of the mass matrix without Majorana terms assures conservation of the total lepton number [14] 16 17, but the individual lepton flavours are violated through the neutrino mixings [16/17/1819]. Through the neutrino mixings, the SM neutrino fields become massless (light) neutrino fields $\left(\nu_{i}\right.$, $i=1,2,3)$ with small admixture of the heavy (quasi-)Dirac fields $\left(N_{i}, i=4, \cdots\right)$. Through the loops, in which only the massive neutrinos give a contribution, the heavy (quasi-)Dirac neutrino components of the SM neutrino field give rise to the LFV effects that are not GIM suppressed. The CKM-type matrix $\left(B_{l_{i} n_{j}}\right)$ which contains neutrino mixing matrix is not known in details. Only the upper limits, $\left(s_{L}^{\nu_{i}}\right)^{2}=\sum_{j=4, \cdots}\left|B_{l_{i} N_{j}}\right|^{2}$ 2021, on the combinations of its elements are limited experimentally 22]. The leading-order LFV contributions come from the loops with two or four $B_{l N}$ matrices (there is one tree-level contribution, but it is negligible compared to the loop contributions). The combinations of matrix elements which appear in the loop contributions to the LFV amplitudes cannot be determined, too. Therefore, for given $\left(s_{L}^{\nu_{i}}\right)^{2}$-s only the upper limits of the LFV amplitudes can be found.

In model B the main sources of LFV are lowest-order loop amplitudes with two or four lepton-slepton-chargino/neutralino vertices [11. The constants in these vertices are defined by mSUGRA SUSY breaking mediation mechanism 12 and the underlying GUT model, here chosen to be the minimal $S O(10)$ model 4567879 . The model contains two $S O(10)$ Higgs superfields, $\mathbf{1 0}$ and $\overline{\mathbf{1 2 6}}$ in the Yukawa sector, and additional Higgs superfields $\mathbf{1 2 6}$ and $\mathbf{2 1 0}$ to preserve the supersymmetry at the GUT scale [23] and to break the $S O(10)$ symmetry to $S U(3)_{c} \times$ $S U(2)_{L} \times U(1)\left(G_{321}\right)$ symmetry 2324]. By assumptions of the mSUGRA mediation mechanism, the soft SUSY breaking terms at the GUT scale (the mass term and the trilinear interaction term) satisfy the universality conditions, according to which the mass soft SUSY- breaking term is flavour-diagonal. In the renormalization group (RG) evolution from the GUT scale to the SM scale, mass soft SUSY-breaking terms receive the off-diagonal LFV contributions through the Dirac-neutrino Yukawa couplings [10. Diagonalization of the sfermion and gaugino mass matrices leads to the chargino and neutralino mass eigenstates with LFV lepton-sleptonchargino/neutralino vertices. Only three parameters, defining the universal soft SUSY-breaking parameters at the GUT scale, have to be introduced in this procedure. Concerning the mixing matrices in model $\mathrm{B}$, which define the mass eigenstates, they are known in details. Therefore, for a given set of initial parameters, LFV amplitudes can be precisely evaluated. That allows to determine both the lower and upper bounds of LFV decay rates [25].

Concerning the RGE procedure there are two subtleties. First, as there are only two Higgs superfields in the Yukawa superpotential, the lepton and quark masses at the GUT scale are defined in terms of two mass matrices. That leads to two equations relating the quark and lepton masses at the GUT scale, which depend on two complex parameters [5]. The quark and lepton masses at the GUT scale are obtained RG-evoluting them from the experimental values at the scale of the $Z$-boson mass. Inserting them into the two equations one can find the solutions for the two complex parameters (the phase of one parameter cannot be determined). With that, fermion mass matrices (and corresponding Yukawa matrices) are determined at the GUT scale, except for the right-neutrino mass matrix. To determine the right-handed neutrino matrix, one more complex parameter has to be introduced [5]. With rightneutrino masses determined, the light neutrino masses can be found using the type I see-saw mechanism. RG-evoluting the light neutrino operator one obtains the light-neutrino mass matrix at the low-energy scale which has to agree with the present neutrino-oscillation data. The agreement can be achieved. In fact, fitting of the neutrino-oscillation data is taken as a necessary condition that has to be fulfilled before proceeding to further calculations. That means that in the LFV breaking couplings, the information 
on the experimental neutrino oscillation data is built in. Second, the $S O(10)$ model predicts the proton decay. The fitting of the model parameters can be achieved to satisfy the present proton decay lower bound [26]. A detailed analysis of the Higgs mass matrices is needed to obtain the parameters defining the proton decay, and RG evolution of these parameters to the proton mass scale is necessary to obtain the the effective proton-decay Lagrangian [2324]. Therefore, in principle, model B can relate branching ratios for neutrinoless LFV decays of charged leptons with the neutrino-oscillation data and lifetime of the proton.

\section{Effective Lagrangians for LFV interac- tions}

In any model containing SM as its low-energy limit, the following effective Lagrangians describe the neutrinoless LFV decays

$$
\begin{aligned}
\mathcal{L}_{\ell_{i} \ell_{j} \gamma}^{\mathrm{eff}}= & -e \bar{\ell}_{j}\left[\left(-\partial^{2} \gamma_{\mu}+\not \partial \partial_{\mu}\right) A^{\mu}\right. \\
& \times\left(\mathcal{P}_{1 \gamma}^{L} P_{L}+\mathcal{P}_{1 \gamma}^{R} P_{R}\right) \\
& \left.+\sigma_{\mu \nu} \partial^{\nu} A^{\mu}\left(\mathcal{P}_{2 \gamma}^{L} P_{L}+\mathcal{P}_{2 \gamma}^{R} P_{R}\right)\right] \ell_{i}, \\
\mathcal{L}_{\ell_{i} \ell_{j} Z}^{\mathrm{eff}}= & g \bar{\ell}_{j}\left[\gamma_{\mu} Z^{\mu}\left(\mathcal{P}_{Z}^{L} P_{L}+\mathcal{P}_{Z}^{R} P_{R}\right)\right] \ell_{i} \\
\mathcal{L}_{\ell_{i} \ell_{j} H}^{\mathrm{eff}}= & g \bar{\ell}_{j}\left[\mathcal{P}_{H}^{L} P_{L}+\mathcal{P}_{H}^{R} P_{R}\right] \ell_{i} H \\
\mathcal{L}_{\mathrm{eff}}^{\mathrm{box}}= & \sum_{\bar{q}_{a} q_{b} X X^{\prime}} \sum_{\Gamma} \sum_{\Gamma a b}^{X X^{\prime}} \\
& \times\left(\bar{\ell}_{j} \Gamma P_{X} \ell_{i}\right)\left(\bar{q}_{a} \Gamma P_{X^{\prime}} q_{b}\right)
\end{aligned}
$$

where $P_{L, R}=\left(1 \pm \gamma_{5}\right) / 2$. The dummy variables of the sums in the last expression assume following values $\bar{q}_{a} q_{b}=\bar{u} u, \bar{d} d, \bar{s} s, \bar{d} s, \bar{s} d, X, X^{\prime}=$ $L L, R R, L R, \Gamma=\gamma_{\mu}, 1, \sigma_{\mu \nu}$. The Lagrangians contain the SM fields only. The effective Lagrangians describe the decays $\ell \rightarrow \ell^{\prime} \gamma^{*}, \ell \rightarrow \ell^{\prime} Z^{*}$, $\ell \rightarrow \ell^{\prime} H^{*}$ and $\ell \rightarrow \ell^{\prime} q_{a} q_{b}$, respectively, where $\ell$ and $\ell^{\prime}, \gamma^{*}, Z^{*}$ and $q_{a}$ and $q_{b}$ denote leptons, photon, Z-boson, Higgs and quarks, respectively (star denotes an off-mass-shell particle). The effective Lagrangian for the decay of lepton into three leptons is not given because we do not consider these decays. They have been considered previously in model A 1819 and B 11. It has almost the same Lorentz structure as the effective Lagrangian for $\ell \rightarrow \ell^{\prime} q_{a} q_{b}$. The informa- tion on LFV induced by a model induces is contained in the form factors $\mathcal{P}_{1,2 \gamma}^{L}, \mathcal{P}_{1,2 \gamma}^{R}, \mathcal{P}_{Z}^{L}, \mathcal{P}_{Z}^{R}$, $\mathcal{P}_{H}^{L}, \mathcal{P}_{H}^{R}, \mathcal{B}_{\Gamma a b}^{X X^{\prime}}$. Form factors contain the loop functions of loop diagrams describing the LFV processes. The structure of the "photon" effective Lagrangian reflects the gauge invariance of the corresponding amplitude. The effective Lagrangian for the "box" process $\ell \rightarrow \ell^{\prime} q_{a} q_{b}$ is the most general one. In many models just few of the form factors are different from zero. Model A contains only terms bilinear in $\gamma_{\mu} P_{L, R}$ matrices, while model $\mathrm{B}$ contains all form factors.

Using the effective Lagrangians the neutrinoless SL LFV, amplitudes of charged leptons and amplitudes for $\ell \rightarrow \ell^{\prime} \gamma$ processes may be found at the quark-lepton level.

\section{Hadronization procedure}

To obtain the amplitudes of neutrinoless SL LFV charged lepton decays in terms of lepton and meson fields, the quark currents have to be transformed into the corresponding meson fields. Here only the decays with one or two pseudoscalar mesons or one vector meson are considered. The conversion of the axial-vector and vector currents into pseudoscalar and vector mesons, respectively is achieved invoking the PCAC hypothesis [27] and VMD (vector-meson dominance) hypothesis 282930, respectively. The scalar and pseudoscalar currents are hadronized comparing the QCD quark Lagrangian with the corresponding effective meson Lagrangian [31. The tensor currents are hadronized using the equations of motion for current quarks 32 and VMD hypothesis. The semileptonic LFV amplitudes for processes with two pseudoscalar mesons in the final state have scalar-quark current contribution and vector-quark current contributions through the vector-meson resonances decaying into two pseudoscalar mesons. For that purpose the Lagrangian of vector-meson-pseudoscalarmeson interactions is introduced 3334 .

\section{Comparison of the branching ratios}

With amplitudes at lepton-meson level, the comparison and evaluation of the decay rates 
of the neutrinoless SL LFV charged lepton decays and $\ell \rightarrow \ell^{\prime} \gamma$ decays can be performed. For comparison of decay rates it is useful to classify them in terms of the basic subamplitudes they contain, $\mathcal{A}_{\gamma}, \mathcal{A}_{Z}, \mathcal{A}_{H}$ and $\mathcal{A}_{b_{l q}}$, which correspond to the effective Lagrangians (11), (2), (3) and (4), respectively. Such a classification is given in Table 1 for the processes considered here.

Table 1

The decay rates for the charged lepton neutrinoless SL LFV processes and $\ell \rightarrow \ell^{\prime} \gamma$ processes.

\begin{tabular}{lll}
\hline \hline process & model A & model B \\
\hline$\ell \rightarrow \ell^{\prime} \gamma$ & $\gamma$ & $\gamma$ \\
$\left(\tau \rightarrow \ell P^{0}\right)_{c}$ & $\gamma, Z, b_{l q}$ & $\gamma, Z, b_{l q}$ \\
$\left(\tau \rightarrow \ell P^{0}\right)_{n}$ & $\ell-q$-box & $b_{l q}$ \\
$\left(\tau \rightarrow \ell V^{0}\right)_{c}$ & $\gamma, Z, b_{l q}$ & $\gamma, Z, b_{l q}$ \\
$\left(\tau \rightarrow \ell V^{0}\right)_{n}$ & $b_{l q}$ & $b_{l q}$ \\
$\left(\tau^{-} \rightarrow \ell^{\prime}-P_{1} P_{2}\right)_{c}$ & $\gamma, Z, H, b_{l q}$ & $\gamma, Z, H, b_{l q}$ \\
$\left(\tau^{-} \rightarrow \ell^{\prime-} P_{1} P_{2}\right)_{n}$ & $b_{l q}, W^{+} W^{-}$ & $b_{l q}$ \\
$\left(\tau^{-} \rightarrow \ell^{\prime-} P_{1} P_{2}\right)_{c, H}$ & $H, W^{+} W^{-}$ & $H$ \\
\hline \hline
\end{tabular}

$\mathrm{c}(\mathrm{n})$ denotes (non)conservation of the quark flavour

In model A there is a window of the heavy neutrino masses $\left(m_{N}\right)$ where the matrix elements $B_{l N}$ are not constrained by heavy neutrino mass $\left(m_{N}\right)$ values. Within that window, the functional dependence of the subamplitudes corresponds to the functional dependence of loop functions constituting the subamplitudes. Speciffically, the subamplitudes $\mathcal{A}_{\gamma}, \mathcal{A}_{Z}, \mathcal{A}_{H}$ and $\mathcal{A}_{b_{l q}}$ behave as $\ln m_{N}, m_{N}^{2}, m_{N}^{2}$ and $\ln m_{N}$, respectively. The $\mathcal{A}_{Z}$ has the largest combination of couplings and if it contributes to a LFV amplitude, it determines its behaviour in the large mass limit. For very large masses, perturbative unitarity bound forces $B_{l N}$ to behave as inverse of the heavy neutrino mass leading to $\ln m_{N} / m_{N}^{2}, 1 / m_{N}^{2}, 1 / m_{N}^{2}$ and $\ln m_{N} / m_{N}^{4}$ behaviour of the amplitudes $\mathcal{A}_{\gamma}$, $\mathcal{A}_{Z}, \mathcal{A}_{H}$ and $\mathcal{A}_{b_{l q}}$ respectively. That behaviour assures that there is no LFV when the heavy neutrino mass tends to infinity. Comparing the expressions for the branching ratios in which only the dominant subamplitude is retained, one finds that there are only two groups of processes that are experimentally interesting, one with $\mathcal{A}_{\gamma}$ only ( $\ell \rightarrow \ell^{\prime} \gamma$ processes), and the other with the
$\mathcal{A}_{Z}$ amplitude (all quark-flavour conserving processes. The processes with box amplitude are strongly suppressed by their $m_{N}$ dependence and couplings in the vertices. The Higgs amplitude is strongly suppresses by the Higgs Yukawa couplings. Within the group with $\mathcal{A}_{\gamma}$ amplitude, by far the best process is $\mu \rightarrow e \gamma$. The branching ratios for the processes with the $\mathcal{A}_{Z}$ amplitude are all of the same order of magnitude, the $\tau \rightarrow \pi^{0}$, and $\tau \rightarrow \rho$ being somewhat better than the other processes.

In model $\mathrm{B}$, the characteristic scale of new physics is the mass of the lightest SUSY particle $m_{\mathrm{SUSY}}$. Not only the loop functions in the $\mathcal{A}_{\gamma}, \mathcal{A}_{Z}$ and $\mathcal{A}_{b_{l q}}$ have the softer behaviour than in model A, $1 / m_{\text {SUSY }}^{2}, \ln m_{\text {SUSY }}$ and $1 / m_{\text {SUSY }}^{2}$ respectively, but all coupling constants decrease with $m_{\mathrm{SUSY}}$ at least as fast as $1 / m_{\mathrm{SUSY}}$. That leads to the dominance of the $\mathcal{A}_{\gamma}$ (photon penguin amplitude) in the large mass limit. Again, there is no LFV in the limit when the characteristic mass of the new physics tends to infinity. All branching ratios decrease as $m_{\mathrm{SUSY}}$ is increased, opposite to the behaviour of branching ratios in model A. We do not discuss the SM Higgs amplitude, because the model B has five Higgs bosons and some of them may have the LFV at the tree level. Limit on the tree-level Higgs contribution constraints combination of the Higgs boson mass and its couplings. In model $\mathrm{B}$, the region of allowed values of mass parameter $m_{\mathrm{SUSY}}$ is small. As explained above, the couplings are precisely known when the parameters of the model are given. Therefore, upper and lower bound for $m_{\mathrm{SUSY}}$ can be determined if there are adequate experimental data. The lower $m_{\text {SUSY }}$ bound $\left(m_{\text {SUSY }}=560 \mathrm{GeV}\right)$ is obtained from the $\mu \rightarrow e \gamma$ process. The upper bound $m_{\mathrm{SUSY}}=800 \mathrm{GeV}$ is determined from the present discrepancy between values of the muon magnetic moment obtained from the $e^{+} e^{-}$data and $\tau$ data [37] which cannot be explained within the SM at the moment. Within so small range of $m_{\text {SUSY }}$ values, it is hard to determine which of the amplitudes dominates without detailed numerical study. The numerical values show that the different processes have different behaviour, with softer $m_{\mathrm{SUSY}}$ for $\ell \rightarrow \ell^{\prime} \gamma$ processes. That indicates that the contributions of some of three con- 
tributions compete for dominance in the branching ratio. Again, among all the processes the $\mu \rightarrow e \gamma$ is the most interesting from the experimental point of view. All numerical results for both model A and B are shown in Table 2. For model B we find that in the parameter region, $560 \mathrm{GeV} \leq m_{\mathrm{SUSY}} \leq 800 \mathrm{GeV}$ consistent with all the experimental data, the predicted values for the semileptonic LFV branching ratios are close to the corresponding current experimental upper bounds. Therefore, they may be tested in the near future.

\section{Acknowledgments}

A.I. would like to thank to I. Picek and S. Fajfer for the discussions on current-quark masses and evaluation of tensor-quark current. A part of the work was presented by A.I. at the workshop, "The 8th International Workshop on TauLepton Physics (Tau04)", held in Nara-ken New Public Hall, Japan. We are grateful to all organizers of this workshop and particularly to Prof. Ohshima for his kind hospitality extended to A.I and T.K. during their stay at Nagoya University. T.F. would like to thank S.T. Petcov for his hospitality at SISSA. This work of T.F. and T.K. was supported by the Grant in Aid for Scientific Research from the Ministry of Education, Science and Culture of Japan and the work of T.K. was supported by the Research Fellowship of the Japan Society for the Promotion of Science (\# 16540269 and \# 7336). The work of A.I was supported by the Ministry of Science and Technology of Republic of Croatia under contract 0119261.

\section{REFERENCES}

1. E. Witten, Nucl. Phys. B 268, 79 (1986).

2. R.N. Mohapatra and J.W.F. Valle, Phys. Rev. D 34, 1642 (1986).

3. M. Dittmar, A. Santamaria, M.C. GonzalezGarcia and J.W.F. Valle, Nucl. Phys. B 332, 1 (1990).

4. K.S. Babu and R.N. Mohapatra, Phys. Rev. Lett. 70, 2845 (1993).

5. T. Fukuyama and N. Okada, JHEP 0211, 011 (2002); K. Matsuda, Y. Koide, T. Fukuyama and H. Nishiura, Phys. Rev. D 65, 033008 (2002), [Erratum-ibid. D 65, 079904 (2002)]; K. Matsuda, Y. Koide and T. Fukuyama, Phys. Rev. D 64, 053015 (2001).

6. H.S. Goh, R.N. Mohapatra and Siew-Phang Ng, Phys. Lett. B 570, 215 (2003); Phys. Rev. D 68, 115008 (2003).

7. B. Bajc, G. Senjanović and F. Vissani, Phys. Rev. Lett. 90, 051802 (2003).

8. B. Dutta, Y. Mimura and R.N. Mohapatra, Phys. Rev. D 69, 115014 (2004).

9. K. Matsuda, Phys. Rev. D 69, 113006 (2004).

10. F. Borzumati, A. Masiero, Phys. Rev. Lett. 57, 961 (1986).

11. J. Hisano, T. Moroi, K. Tobe and M. Yamaguchi, Phys. Rev. D 53, 2442 (1996).

12. R. Barbieri, S. Ferrara and C.A. Savoy, Phys. Lett. B 119, 343 (1982); A.H. Chamseddine, R. Arnowitt and P. Nath, Phys. Rev. Lett. 49, 970 (1982); L.J. Hall, J. Lykken and S. Weinberg, Phys. Rev. D 27, 2359 (1983).

13. Y. Enari [Belle collaboration], talk given at this workshop, http://www.hepl.phys nagoya-u.ac.jp/ public/Tau04/].

14. J.W.F. Valle, in NUCLEAR BETA DECAYS AND NEUTRINO: proceedings. Edited by T. Kotani, H. Ejiri, E. Takasugi. Singapore, World Scientific, 1986. 542p.

15. M.C. Gonzalez-Garcia and J.W.F. Valle, Phys. Lett. B 216 (1989).

16. J. Bernabéu, A. Santamaria, J. Vidal, A. Mendez and J.W.F. Valle, Phys. Lett. B 187, 303 (1997).

17. N. Riuz and J.W.F. Valle, Phys. Lett. B 246, 249 (1990).

18. M.C. Gonzalez-Garcia and J.W.F. Valle, Mod. Phys. Lett. A 7, 477 (1992).

19. A. Ilakovac and A. Pilaftsis, Nucl. Phys. B 437, 491 (1995).

20. P. Langacker and D. London, Phys. Rev. 38 (1988) 886; E. Bhattachatyya, A. Datta, S.N. Ganguli and A. Raychaudhuri, Mod. Phys. Lett A 6, 2991 (1991); E. Nardi, E. Roulet and D. Tommasini, Nucl. Phys. B 386, 239 (1992).

21. D. Tommasini, G. Barneboim, J. Bernabéu and C. Jarlskog, Nucl. Phys. B 444, 451 (1995). 
Table 2

The branching ratios for neutrinoless SL LFV of a charged lepton and and $\ell \rightarrow \ell^{\prime} \gamma$ processes.

\begin{tabular}{llll}
\hline \hline decay modes & exp. bound & model A & model B \\
\hline \hline$\mu \rightarrow e^{-} \gamma$ & $1.2 \times 10^{-11}$ & $8.1 \times 10^{-9} x_{\mu e}^{2}$ & $1.2 \times 10^{-11}$ \\
$\tau \rightarrow e^{-} \gamma$ & $3.8 \times 10^{-7}$ & $3.4 \times 10^{-8} x_{\tau e}^{2}$ & $\left(2.6 \times 10^{-10}, 1.0 \times 10^{-9}\right)$ \\
$\tau \rightarrow \mu^{-} \gamma$ & $3.1 \times 10^{-7}$ & $6.7 \times 10^{-9} x_{\tau \mu}^{2}$ & $\left(1.1 \times 10^{-9}, 4.5 \times 10^{-9}\right)$ \\
\hline$\tau \rightarrow e^{-} \pi^{0}$ & $1.9 \times 10^{-7}$ & $2.8 \times 10^{-6} y_{\tau e}^{2}$ & $\left(7.4 \times 10^{-9}, 4.1 \times 10^{-8}\right)$ \\
$\tau \rightarrow \mu^{-} \pi^{0}$ & $4.3 \times 10^{-7}$ & $5.4 \times 10^{-7} y_{\tau \mu}^{2}$ & $\left(3.4 \times 10^{-8}, 1.8 \times 10^{-7}\right)$ \\
$\tau \rightarrow e^{-} \eta$ & $2.3 \times 10^{-7}$ & $4.0 \times 10^{-7} y_{\tau e}^{2}$ & $\left(1.1 \times 10^{-9}, 6.2 \times 10^{-9}\right)$ \\
$\tau \rightarrow \mu^{-} \eta$ & $1.3 \times 10^{-7}$ & $7.8 \times 10^{-8} y_{\tau \mu}^{2}$ & $\left(5.1 \times 10^{-9}, 1.3 \times 10^{-8}\right)$ \\
$\tau \rightarrow e^{-} \rho^{0}$ & $2.0 \times 10^{-6}$ & $2.7 \times 10^{-6} y_{\tau e}^{2}$ & $\left(4.0 \times 10^{-14}, 2.9 \times 10^{-14}\right)$ \\
$\tau \rightarrow \mu^{-} \rho^{0}$ & $6.3 \times 10^{-6}$ & $5.3 \times 10^{-7} y_{\tau \mu}^{2}$ & $\left(1.3 \times 10^{-13}, 1.2 \times 10^{-12}\right)$ \\
$\tau \rightarrow e^{-} \phi$ & $6.9 \times 10^{-6}$ & $2.7 \times 10^{-6} y_{\tau e}^{2}$ & $\left(9.6 \times 10^{-10}, 5.1 \times 10^{-9}\right)$ \\
$\tau \rightarrow \mu^{-} \phi$ & $7.0 \times 10^{-6}$ & $5.3 \times 10^{-7} y_{\tau \mu}^{2}$ & $\left(4.1 \times 10^{-9}, 2.2 \times 10^{-8}\right)$ \\
\hline$\tau \rightarrow e^{-} \pi^{+} \pi^{-}$ & $2.2 \times 10^{-6}$ & $2.7 \times 10^{-6} y_{\tau e}^{2}$ & \\
$\tau \rightarrow \mu^{-} \pi^{+} \pi^{-}$ & $8.2 \times 10^{-6}$ & $5.2 \times 10^{-7} y_{\tau \mu}^{2}$ & \\
$\tau \rightarrow e^{-} K^{+} K^{-}$ & $6.0 \times 10^{-6}$ & $1.1 \times 10^{-6} y_{\tau e}^{2}$ & \\
$\tau \rightarrow \mu^{-} K^{+} K^{-}$ & $1.5 \times 10^{-5}$ & $2.1 \times 10^{-7} y_{\tau \mu}^{2}$ & \\
\hline \hline
\end{tabular}

The model A values are upper limits of branching ratios multiplied by combinations of $B_{l N}$ matrix elements that are always smaller than one [36. For model B the first(second) value in brackets represents lower(upper) bound for a branching ratio. For $\mu \rightarrow e \gamma$ only the upper bound is given, which served as an input parameter to determine the minimal $m_{S U S Y}$ value.

22. E. Nardi, E. Roulet and D, Tommasini, Phys. Lett. B 327, 319 (1994).

23. T. Fukuyama, A. Ilakovac, T. Kikuchi, S. Meljanac and N. Okada, hep-ph/0401213

24. T. Fukuyama, A. Ilakovac, T. Kikuchi, S. Meljanac and N. Okada, to be published in J. Math. Phys.

25. T. Fukuyama, A. Ilakovac, T. Kikuchi, in preparation.

26. T. Fukuyama, A. Ilakovac, T. Kikuchi, S. Meljanac and N. Okada, JHEP 0409 (2004) 052. hep-ph/0401213.

27. R.E. Marshak, Riazuddin and C.P. Ryan, Weak Interactions in Particle Physics, (Wiley, New York, 1969).

28. J.J. Sakurai, Currents and Mesons, (University of Chicago Press, Chicago, 1969).

29. V. de Alfaro, S. Fubini and C. Rossetti, Currents in Hadron Physics, (North-Holland, Amsterdam, 1973).

30. M. Zielinski, Acta Phys. Polon. B 18, 455
(1987).

31. W. A. Bardeen, A. J. Buras, and J.-M. Gérard, Nucl. Phys. B 293, 787 (1987); W. A. Bardeen, A. J. Buras, and J.-M. Gérard, Phys. Lett. B 180, 133 (1986); R. S. Chivukula, J.M. Flynn and H. Georgy, ibid. 171, 453 (1986).

32. J. Gasser and H. Leutwyler, Ann. Phys. 158, 142 (1994).

33. M. Bando, T. Kugo, K. Yamawaki, Phys. Rep. 164, 217 (1988).

34. M. Bando, T. Kugo, K. Yamawaki, Nucl. Phys. B 259, 493 (1985).

35. S. Fajfer and A. Ilakovac, Phys. Rev. D 57, 4219 (1998).

36. A. Ilakovac, Phys. Rev. D 62, 036010 (2000).

37. G. W. Bennett et al. [Muon g-2 Collaboration], Phys. Rev. Lett. 89, 101804 (2002) [Erratum-ibid. 89, 129903 (2002)] arXiv:hep-ex/0208001. 